\title{
Patient education programs - can they improve outcomes in COPD?
}

\author{
Melanie Harris' \\ Brian J Smith ${ }^{2,3}$ \\ Antony Veale ${ }^{4}$ \\ 'Department of Medicine, The \\ University of Adelaide, Australia; \\ ${ }^{2}$ Clinical Epidemiology Unit and \\ Respiratory Medicine and ${ }^{3}$ Sleep \\ Disorders Unit, The Queen Elizabeth \\ Hospital and Health Services, \\ Adelaide, Australia; ${ }^{4}$ The Queen \\ Elizabeth Hospital and Health \\ Services, Adelaide, Australia
}

\begin{abstract}
It is important to assess the effectiveness of patient education programs for people with COPD (chronic obstructive pulmonary disease) to ensure that limited health resources are being spent effectively. We aimed to assess the effectiveness of programs reported to date, and to look for ways of designing more effective programs.

COPD patient education to date has produced little demonstrated success, but studies, education programs and study reports all show limitations. To demonstrate links between outcomes and patient education program components, there is a need for more trials which combine process and outcome evaluation. Programs to date have relied too heavily on the provision of medical information to patients. Programs which also aim to improve disease management self-efficacy hold promise but further determinants of health behavior should be included also, as part of more systematic program design. Program components need to be clearly described and the rationale for their use justified in trial reports. This will produce an evidence base that should show what role education programs can play in improving outcomes, and inform the development of more effective programs.
\end{abstract}

Keywords: patient education, educational design, research design

\section{Introduction}

Patient education programs to support patient participation in disease management have been proposed as an important strategy in limiting the growing burden of chronic disease (Lorig and Holman 2003). These programs have been studied for many years for chronic diseases such as diabetes, arthritis and asthma (Warsi et al 2004) and more recently for COPD (chronic obstructive pulmonary disease) (Monninkhof et al 2003). Reported success varies between conditions (Warsi et al 2004) so it is important to assess the effectiveness of programmes specifically in COPD.

Our review aims to answer the following questions:

1. Have COPD patient education programs reported to date led to improved outcomes?

2. Can we design more effective programs?

COPD patient and caregiver education is a component of many successful pulmonary rehabilitation programs. As it is not easy to separate out the effects of education (Lacasse et al 1997) we chose to review only stand-alone educational programs. We also focus our review on programs with educational content that covers multiple topics of potential relevance to COPD patients, rather than single areas of therapy such as diet, or inhaler technique, or use of oxygen delivery devices.

Correspondence: Melanie Harris

Clinical Epidemiology and Health

Outcomes Unit, DX465 I54, Basil Hetzel Institute, The Queen Elizabeth Hospital and Health Services, 28 Woodville Road, Woodville South, SA 50I I, Australia

Tel +620882227933

Fax +620882226896

Email melanie.harris@nwahs.sa.gov.au

\section{Have COPD patient education programs led to improved outcomes?}

Most studies included in a Cochrane review of self-management education for COPD (Monninkhof et al 2003) demonstrated no benefits. The review included 
eight studies, published between 1985 and 2000, which compared self-management education for COPD with usual care. Group or individual education sessions were the most common type of intervention, occupying less than one hour to 26 hours. Numbers of participants randomized ranged from 50 to 659 and follow up durations from 2- to 12 -months. Studies reported a range of outcomes. No effects were found on hospital admissions, emergency visits, days lost from work or lung function. Results for quality of life measures, visits to health professionals and symptoms were described as inconclusive. One reviewed trial measured the use of short-acting beta-2-agonist rescue medication and found that it was reduced for participants who had attended education. Three studies assessed use of corticosteroids and two assessed use of antibiotics for respiratory symptoms. Both corticosteroids and antibiotics were used significantly more for participants who had attended education. Reviewers suggested that this might indicate increased help seeking (Monninkhof et al 2003).

Results from these studies may suggest that some types of patient education can lead to earlier intervention for exacerbations. However, limited success and study limitations meant that Cochrane reviewers were unable to form practice recommendations. Instead, they called for standardized trial outcomes, and for studies evaluating action plans and theory-based behavior-change programs with core elements described (Monninkhof et al 2003).

Three studies published since 2001 have also produced mixed results. One randomized trial $(n=191)$ (Bourbeau et al 2003) evaluated a program which included 7-8 hours of one-to-one teaching and a customized action plan for exacerbations. The trial demonstrated no effect on lung function, exacerbation rate or overall COPD-related quality of life after 12-months, but a reduction in emergency department visits, unscheduled doctor visits and admissions. As the intervention also included contact with a case manager for the duration of the study, outcomes could not definitely be attributed to the education program. Another randomized trial (Monninkhof et al 2003) ( $n=248)$ evaluated a program comprising 10 hours of skill-oriented education and a fitness program which continued weekly for 12-months. The study demonstrated no improvement in quality of life, six-minute walk, symptoms or self-confidence and found a higher rate of self-reported exacerbations in the intervention group. A further randomized trial (Hesselink et al 2004) $(n=276)$ evaluated a 1-2 hour one-to-one teaching program which aimed to improve health outcomes by improving patient knowledge and self-care skills. The trial found no effect after 12-months and 24-months on symptoms, quality of life, compliance, coping, self-efficacy or smoking cessation, with inhaler technique the only measure showing improvement.

Overall, patient education has shown success only in some studies and this success appears to be confined to earlier intervention, aimed at reducing exacerbations. It would be useful to identify program characteristics associated with this success. Unfortunately, program descriptions in trial reports are very similar and focus on topics covered and methods of information delivery rather than links with any underlying behavior-change rationale. Particular characteristics or theoretical bases of more successful interventions cannot therefore be identified.

\section{Can we design more effective programs?}

For more consistent success we need to identify and replicate particular components of the educational programs which have led to improved outcomes. For outcomes which have so far been unaffected by patient education, we need to look for new program designs.

\section{Identifying program components associated with success}

As well as evaluating the success of an overall educational program, research designs should demonstrate how particular components contribute to the success or failure of the whole.

The success or failure of individual program components can be identified when trials include process as well as outcome evaluations (Hulscher et al 2003). Process evaluations have rarely been included in patient education trials (Cooper et al 2001) but two recent examples show their usefulness for COPD patient education. A process evaluation was conducted with a trial of patient education plus contact with health professionals which succeeded in reducing hospital admissions (Bourbeau et al 2004). The process evaluation demonstrated that participants frequently used four disease-related skills which had been taught (energy conservation, use of antibiotic and prednisolone according to action plan, pursed lip breathing and regular home exercise). Barriers to practicing the skills were also revealed, showing researchers where improvements could be made (Bourbeau et al 2004). Even when overall program outcomes are not achieved, process evaluations can reveal partial successes which can aid further research. For an education and exercise program which produced no change in quality of life, the primary outcome, a process investigation showed patient-perceived benefits not detected by the trial 
These included increased exercise capacity, skills in energy conservation and intention to self treat exacerbations with antibiotics and prednisone (Monninkhof et al 2004).

Educational programs and their rationale now need to be better described so that these successes can be attributed to program components (Cooper et al 2001).

\section{Designing new programs which are likely to succeed}

Early COPD patient education appeared to be based on a premise that improving patients' medical knowledge would improve outcomes. More recently, disease management self-efficacy has been proposed as an alternative or additional determinant of health outcomes. We examine the evidence relating to knowledge and disease management self-efficacy and provide a method for systematic program development based on these and other determinants of health outcomes.

\section{Knowledge}

Most COPD patient education to date has presented medical information to patients, with no behavioral theory underlying program delivery (Monninkhof et al 2003; Bourbeau et al 2004). Patient education has aimed to "transfer knowledge" (Monninkhof et al 2003) about COPD from health professionals to patients, with the implicit expectation that patients would then manage their disease differently and that health outcomes would then improve. Patient knowledge is not always measured in trials of patient education but in four trials knowledge was measured and was found to increase (Bourbeau et al 2004). However, patient outcomes were improved in only one of these trials (Bourbeau et al 2004) suggesting that other patient factors need to be changed for improved outcomes.

\section{Self-efficacy}

Patient self-efficacy, their sense of competence or ability in disease management, is now being advocated as the route to improved outcomes (Monninkhof et al 2003; Bourbeau et al 2004). Bandura's behavior change theory, social cognitive theory (Bandura 2004) positions self-efficacy as the major determinant of behavior change and patient education based on this theory aims to improve disease related self-efficacy (Bodenheimer et al 2002; Lorig and Holman 2003). However, a COPD program which aimed to improve self-efficacy (Bourbeau et al 2004) achieved some but not all of its aims (Bourbeau et al 2003), and, more generally, chronic disease self management programs which claim to be theory based have been no more effective than other programs (Warsi et al 2004). These results may be explained by failure of the theory, poor implementation of the theory in the programs (Wagner et al 1999), or failure to recognize and deal with additional factors required for patient behavior change. While basing education on self-efficacy may assist in improving outcomes, it is likely that other behavioral determinants (Armitage and Conner 2000; Fishbein et al 2001) should be addressed also.

\section{Systematic program development}

Recent advice on complex interventions (Campbell et al 2000; van Bokhoven et al 2003; Michie and Abraham 2004) provides a useful framework for identifying and addressing the range of factors governing patient behavior. This framework requires detailed statements of all behavioral goals, research into mechanisms, barriers and facilitators for desired behaviors, consideration of the full range of psychosocial theories and existing research which could underpin each component of the program, and testing and modification of individual components before trials of the complete intervention. Rigorous processes such as this produce interventions which are more likely to succeed than intuitive poorly-founded designs (Campbell et al 2000; van Bokhoven et al 2003; Michie and Abraham 2004).

\section{Conclusion}

In conclusion, while patient education to date has rarely produced the desired outcomes, there is great potential for improvement in the design of both patient education programs and trials of their effectiveness. Outcomes which can realistically be expected from patient behavior changes must be identified. The susceptibility of the particular behavior to change must be assessed, and the effectiveness of various theory-based educational techniques in bringing about this change. When programs developed in this way have been trialed we will know more definitively what role COPD patient education can play.

\section{References}

Armitage CJ, Conner M. 2000. Social cognition models and health behaviour: a structured review. Psychol Health, 15:173-89.

Bandura A. 2004. Health promotion by social cognitive means. Health Educ Behav, 31:143-64.

Bodenheimer T, Lorig K, Holman H, et al. 2002. Patient self-management of chronic disease in primary care. JAMA, 288:2469-75.

Bourbeau J, Julien M, Maltais F, et al. 2003. Reduction of hospital utilization in patients with chronic obstructive pulmonary disease: a disease-specific self-management intervention. Arch Intern Med, 163:585-91.

Bourbeau J, Nault D, Dang-Tan T. 2004. Self-management and behaviour modification in COPD. Patient Educ Couns, 52:271-7. 
Campbell M, Fitzpatrick R, Haines A, et al. 2000. Framework for design and evaluation of complex interventions to improve health. $B M J$, 321:694-6.

Cooper H, Booth K, Fear S, et al. 2001. Chronic disease patient education: lessons from meta-analyses. Patient Educ Couns, 44:107-17.

Fishbein M, Triandis HC, Kanfer FH, et al. 2001. Factors influencing behavior and behavior change. In: Baum A, Revenson TA, Singer JE eds. Handbook of health psychology. Mahwah, New Jersey: Lawrence Erlbaum. p 3-17.

Hesselink AE, Penninx BW, van der Windt DA, et al. 2004. Effectiveness of an education programme by a general practice assistant for asthma and COPD patients: results from a randomised controlled trial. Patient Educ Couns, 55:121-8.

Hulscher ME, Laurant MG, Grol RP. 2003. Process evaluation on quality improvement interventions. Qual Saf Health Care, 12:40-6.

Lacasse Y, Guyatt GH, Goldstein RS. 1997. The components of a respiratory rehabilitation program: a systematic overview. Chest, 111:1077-88.

Lorig KR, Holman H. 2003. Self-management education: history, definition, outcomes, and mechanisms. Ann Behav Med, 26:1-7.

Michie S, Abraham C. 2004. Interventions to change health behaviours: evidence-based or evidence-inspired? Psychol Health, 19:29-49.
Monninkhof E, van der Valk P, van der Palen J, et al. 2003. Effects of a comprehensive self-management programme in patients with chronic obstructive pulmonary disease. Eur Respir J, 22:815-20.

MonninkhofEM, van der Valk PD, van der Palen J, et al. 2003. Self-management education for chronic obstructive pulmonary disease. Cochrane Database of Syst Rev, CD002990.

Monninkhof E, van der Aa M, van der Valk P, et al. 2004. A qualitative evaluation of a comprehensive self-management programme for COPD patients: effectiveness from the patients' perspective. Patient Educ Couns, 55:177-84.

van Bokhoven MA, Kok G, van der Weijden T. 2003. Designing a quality improvement intervention: a systematic approach. Qual Saf Health Care, 12:215-20.

Wagner EH, Davis C, Schaefer J, et al. 1999. A survey of leading chronic disease management programs: are they consistent with the literature? Manag Care Q, 7:56-66.

Warsi A, Wang PS, LaValley MP, et al. 2004. Self-management education programs in chronic disease: a systematic review and methodological critique of the literature. Arch Intern Med, 164:1641-9. 